\title{
Favorable Prognostic Impact of Cathepsin H (CTSH) High Expression in Thyroid Carcinoma
}

\author{
Pai Peng' \\ Jiang-Yuan Chen ${ }^{2}$ \\ Kai Zheng ${ }^{3}$ \\ Chao-Hua Hu' \\ Yun-Tao Han' \\ 'Department of Breast and Thyroid \\ Surgery, Xiaogan Central \\ Hospital\&Xiaogan Hospital Affiliated to \\ Wuhan University of Science and \\ Technology, Xiaogan, 432000, People's \\ Republic of China; ${ }^{2}$ School of Medicine, \\ Jianghan University, Wuhan, 430000, \\ People's Republic of China; ${ }^{3}$ School of \\ Medicine, Wuhan University of Science \\ and Technology, Wuhan, 430000, People's \\ Republic of China
}

Correspondence: Chao-Hua Hu Department of Breast and Thyroid Surgery, Xiaogan Central Hospital\&Xiaogan Hospital Affiliated to Wuhan University of Science and Technology, Xiaogan, 432000, People's Republic of China

Email huchaohua2006@I26.com
Background: Presently, no study reported the function of cathepsin H (CTSH) in thyroid carcinoma (THCA). The aim of present study was to initially explore the factors affecting CTSH expression, and association between CTSH expression and survival rate in THCA.

Methods: We explored mRNA expression of CTSH in normal and BRCA tissues, and evaluated prognostic impact of CTSH expression on the overall survival of THCA patients. Then, related factors influencing CTSH mRNA expression in THCA were analyzed. Functional enrichment analysis was performed to reveal the potential function of CTSH involved in THCA. We also constructed PPI network among co-expressed genes of CTSH to determine hub genes, followed by association analysis on hub genes with CTSH.

Results: (1) CTSH mRNA was highly expressed in THCA compared with normal group $(P<0.001)$. High expression of CTSH was conducive to the overall survival of THCA patients $(P=0.0027)$. CTSH was then determined as an independent prognostic factor in THCA $(P=0.024)$. (2) The mRNA expression of CTSH was statistically related to patient's histological type, $\mathrm{N}$ stage, $\mathrm{T}$ stage, tumor stage and sample type (all $P<0.001$ ). CTSH copy number variation and methylation also influenced its mRNA expression (all $P<0.001$ ). (3) Pathway analysis indicated that CTSH mainly participated in cancer-related pathways, such as hedgehog signaling pathway, cytokine-cytokine receptor interaction and JAK-STAT signaling pathway (all $P<0.05$ ). (4) The top 10 co-expressed genes in whole PPI network showed significant correlation with CTSH expression (all $P<0.001$ ).

Conclusion: CTSH higher expression was observed in THCA, which caused a good prognosis of patients. CTSH expression might be regulated by multiple factors including clinical characteristic, methylation, copy number and other genes. This study demonstrated the clinical significance of CTSH in THCA, as well as revealed the potential pathway associated with CTSH involved in thyroid cancer.

Keywords: cathepsin $\mathrm{H}$, thyroid carcinoma, high expression, prognosis

\section{Introduction}

The incidence of thyroid carcinoma (THCA) was growing the fastest among all malignancies worldwide, particularly in women. ${ }^{1,2}$ Financial pressure commonly appeared in thyroid cancer than other malignancies. ${ }^{3}$ Largely due to the treatability and favorable survival, THCA has been considered as a "good cancer" which permeated the medical culture. ${ }^{4}$ However, many patients with well-differentiated THCA suffered a persistent and severe decrease in quality of life, lived with fear of recurrences, and commonly experienced feelings of isolation. ${ }^{3}$ But should not be ignored was that cancer metastasis and proliferation remained as the primary causes of poor prognosis and death in THCA patients. ${ }^{5}$ Thus, there was a compelling need 
to explore the mechanisms underlying pathogenesis of THCA, improved the prognosis and life quality of THCA patients.

Cathepsins are lysosomal proteolytic enzymes, which function as cysteine proteinase and play an important role in the catabolism of proteins. ${ }^{6}$ The potent proteolytic activity of cathepsins is known to affect the fate of many intracellular as well as extracellular targets, and deregulation of these functions is associated with tumor proliferation, growth and metastasis. ${ }^{7}$ Extracellular cathepsins were commonly believed to be of central importance in cancer progression by modulating the architecture of the extracellular matrix, intracellular cathepsins have been established as being of extreme significance in mediating cell death through apoptosis. ${ }^{8}$ The therapeutic-targeting potential of cathepsins and their use as reliable prognostic markers has been reported. ${ }^{9}$ Among all the cathepsins, cathepsin H (CTSH) was far less studied and observed in tissues of primary and metastatic tumors in many cancer types. Abnormal expression of CTSH has been found in colorectal carcinoma, ${ }^{10}$ breast cancer $^{11}$ and prostate cancer, $^{12}$ and over expression of CTSH in these cancers was associated with more aggressive disease. Conversely, the patients with head and neck cancer showed a lower level of CTSH than the normal tissue. ${ }^{13}$ Despite the wellknown association between CTSH expression and progression in various cancers, the underlying role of $\mathrm{CTSH}$ in THCA remained unclear.

The present study was conducted to investigate the expression and prognostic value of CTSH in THCA. We found that CTSH was over-expressed in THCA, and high expression was associated with the favorable overall survival. We also explored the possible mechanisms of CTSH in THCA, and provided the potential therapeutic strategies for the treatment of THCA.

\section{Methods}

\section{Impact Analysis of CTSH mRNA} Expression on Prognosis of Patients

The mRNA expression of CTSH in normal and THCA patients were firstly analyzed in GSCA database (http:// bioinfo.life.hust.edu.cn/GSCA). The impact of CTSH expression on overall survival of THCA patients was then evaluated in Kaplan-Meier plotter database (http://kmplot.com/). A restrict 10-year survival analysis on CTSH was also performed based on gender and cancer stages. In addition, we took clinical characteristic, immune cell and tumor purity into consideration, and conducted Cox regression analysis to predict the independent prognostic value of CTSH in THCA.

\section{Association Analysis Between CTSH mRNA Expression and Related Factors}

Association between CTSH mRNA expression and clinical data of THCA patients were firstly evaluated in Mexpress database (https://mexpress.be/). A total of 13 parameters were enrolled in the association analysis, including gender, age at initial pathologic diagnosis, metastatic site, neoplasm depth, neoplasm length, neoplasm width, pathologic primary tumor $(\mathrm{T})$, pathologic regional lymph nodes $(\mathrm{N})$, pathologic distant metastasis (M), new tumor event after initial treatment, histological type, person lifetime risk radiation exposure indicator, person neoplasm cancer status, tumor stage, sample type and copy number. Subsequently, CTSH mRNA expression was compared in terms of significant parameters with statistical significance. In addition, the effects of CTSH methylation and CNV (Copy Number Variation) on the mRNA expression and overall survival of patients were assessed in GSCA database. Correlation between CTSH mRNA expression and abundance of immune cells was also explored in TISIDB database (http://cis.hku.hk/TISIDB/).

\section{CTSH mRNA and Protein Expression in Normal and Cancer Tissues}

The Human Protein Atlas (HPA) database (https://www. proteinatlas.org/) is a Swedish-based program with the aim to map all the human proteins in cells, tissues and organs using integration of various omics technologies, including antibody-based imaging, mass spectrometry-based proteomics, transcriptomics and systems biology. The mRNA and protein expressions of CTSH in normal and human cancer tissues were first explored through HPA database.

\section{Genetic Alteration of CTSH in THCA}

The gene mutation played an important role in the cancer progression. The genetic alteration of CTSH in THCA was explored through Thyroid Carcinoma (TCGA, Firehose Legacy) dataset $(n=397)$ in cBioportal database (http:// www.cbioportal.org/) to obtain the mutation frequency and sites. The mutation types and nucleotide mutation of CTHS in THCA was subsequently assessed in Catalogue Of Somatic Mutations In Cancer (COSMIC) database (https:// cancer.sanger.ac.uk/cosmic). COSMIC was the world's 
A

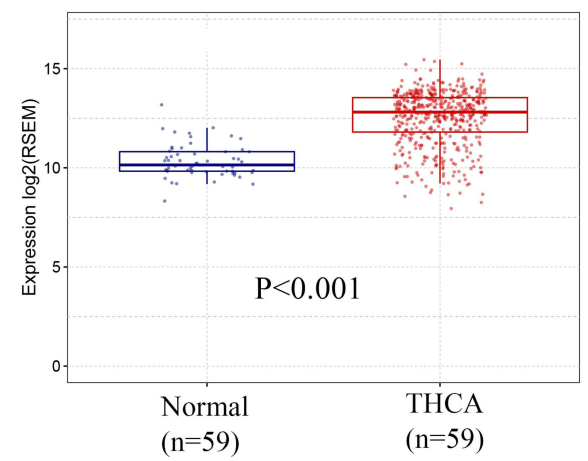

B

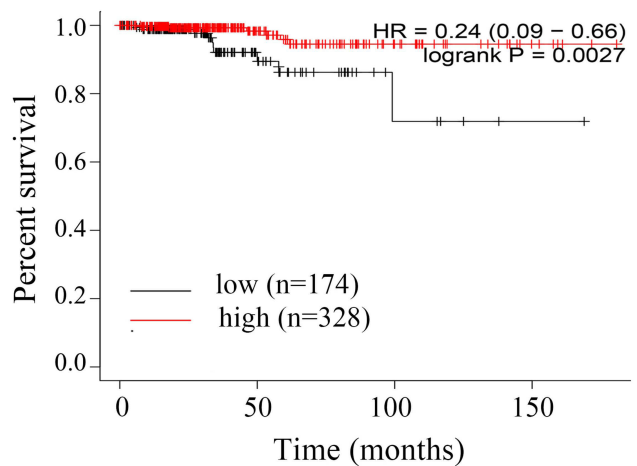

Figure I CTSH mRNA expression analysis. (A) Differential expression of CTSH mRNA in normal and THCA tissues. The independent sample T-test was used to compare difference. (B) Impact of CTSH expression on the overall survival of THCA patients between CTSH high (red) and low expression (black) groups. Survival time was compared with Kaplan-Meier and logrank test.

largest and most comprehensive resource for exploring the impact of somatic mutations in human cancer.

\section{Enrichment Analysis}

For better understanding the role of CTSH in THCA, we got some co-expressed genes playing similar function with $\mathrm{CTSH}$ from cBioportal database. The 207 co-expressed genes with $P<0.001$ and absolute correlation coefficient $>0.6$ were selected for the further enrichment analysis. Functional enrichment analyses were performed in Metascape database (https://metascape.org/) for GO annotation and WebGestalt (http://www.webgestalt.org/) database for KEGG pathway exploration. Additionally, GSEA analysis was performed to explore significant pathways associated with $\mathrm{CTSH}$ involved in THCA. The terms with nominal $P$-value $<0.05$ and FDR $<$ $25 \%$ were considered as significant events.

We also constructed protein-protein interaction (PPI) network of 207 co-expressed genes through String database (https://string-db.org/), and visualized the PPI network by Cytoscape. Among the whole PPI network, we determined the top 10 hub genes using MCC gene ranking methods. Expression correlation between CTSH and 10 hub genes was then evaluated involved in THCA by adjusting the patient's age.

\section{Statistical Analysis}

Related statistical analyses were performed with SPSS 22.0. Independent $t$-test was used for comparisons between two groups, and comparisons between more than three groups were conducted using ANOVA with a post hoc multiple comparison. Survival outcomes including overall survival was compared by Kaplan-Meier analysis and logrank test. Spearman correlation analysis was used to present relationship between 2 variables. $P$ value less than 0.05 was considered as statistically significant.

\section{Results}

\section{Impact of CTSH mRNA Expression on the Prognosis of THCA Patients}

Differential expression analysis showed that expression of CTSH mRNA in THCA was higher than that in normal group (Figure 1A), and difference presented statistical significance $(P<0.001)$. Survival analysis indicated that CTSH mRNA high expression prolonged the overall survival time of THCA patients (Figure 1B) and caused a favorable prognosis $(\mathrm{HR}=0.24, P=0.0027)$.

Restrict analysis on the patient's survival (Table 1) indicated that the survival difference between high and low expression groups was statistically significant both in female $(P=0.0300)$ and male $(P=0.0008)$ patients. The survival difference between two groups was also observed in patients with stage $1(P=0.0051)$ and stage $3(P=0.0078)$. Taking the clinical characteristics and immune cells into consideration,

Table I Restrict Survival Analysis in THCA Patients

\begin{tabular}{|l|l|l|l|}
\hline Subtypes & & HR (95\% CI) & Log Rank $P$ \\
\hline Gender & Female $(\mathrm{n}=367)$ & $0.27(0.08-0.96)$ & 0.0300 \\
& Male $(\mathrm{n}=\mathrm{I35})$ & $0.09(0.02-0.5 \mathrm{I})$ & 0.0008 \\
\hline \multirow{2}{*}{ Cancer stages } & I $(\mathrm{n}=28 \mathrm{I})$ & $0(0-\operatorname{Inf})$ & $0.005 \mathrm{I}$ \\
& II $(\mathrm{n}=52)$ & $0(0-\operatorname{Inf})$ & 0.0720 \\
& III $(\mathrm{n}=\mathrm{II})$ & $0(0-\operatorname{Inf})$ & 0.0078 \\
& IV $(\mathrm{n}=55)$ & $0(0-\operatorname{Inf})$ & 0.2200 \\
\hline
\end{tabular}

Note: The survival time was compared with Kaplan-Meier and log rank test. Abbreviations: $\mathrm{HR}$, hazard ratio; $\mathrm{Cl}$, confidence interval. 
Table 2 Multivariate Cox Regression Analysis on Thyroid Carcinoma Patient $(\mathrm{n}=48 \mathrm{I})$

\begin{tabular}{|l|l|l|l|}
\hline Parameters & Coef & HR (95\% CI) & P-value \\
\hline Age & 0.210 & $1.234(1.131,1.347)$ & $<0.00 I$ \\
Gender-male & -0.195 & $0.823(0.171,3.968)$ & 0.808 \\
Stage 2 & 0.337 & $1.40 I(0.166, I .180 \mathrm{E}-0 \mathrm{I})$ & 0.757 \\
Stage 3 & 0.582 & $1.789(0.259,1.235 \mathrm{E}-0 \mathrm{I})$ & 0.555 \\
Stage 4 & 3.383 & $29.450(2.357,3.680 \mathrm{E}-02)$ & 0.009 \\
Purity & 7.277 & $1447.172(33.044,6.337 \mathrm{E}-04)$ & $<0.00 \mathrm{I}$ \\
B cell & 5.924 & $373.752(0.004,3.675 \mathrm{E}-07)$ & 0.313 \\
CD4 T cell & 10.290 & $29,428.680(1.077,8.044 \mathrm{E}-08)$ & 0.048 \\
Macrophage & -30.190 & $0.000(0.000,0.565)$ & 0.046 \\
CTSH & -0.714 & $0.490(0.263,0.911)$ & 0.024 \\
\hline
\end{tabular}

Note: Multiple selection available: female is the reference level for gender, while stage I for stage as well.

Abbreviations: coef, correlation coefficient; $\mathrm{HR}$, hazard ratio. $\mathrm{Cl}$, confidence interval.

we performed multivariate cox regression analysis to predict the independent prognostic factors of THCA. The results (Table 2) indicated that parameters of age, stage 4, purity, CD4 T cell, macrophage and CTSH were independent factors for THCA prognosis. It followed that CTSH expression was associated with survival of THCA patients as well as determined as an independent prognostic factor, suggesting the clinical significance of CTSH in THCA.

\section{Association Between CTSH mRNA Expression and Related Factors}

Due to the significance of CTSH mRNA expression on the survival of THCA patients, we then explored the related clinical characteristics of THCA patients affecting CTSH mRNA expression level (Figure 2). Among 13 phenotypes, we found that CTSH mRNA expression was statistically related to 6 parameters, including histological type, pathologic-N, pathologic-T, tumor stage, sample type and copy number (all $P<0.001$ ). Remaining parameters showed no statistical correlation with CTSH mRNA expression.

We subsequently explored the detailed expression of CTSH mRNA expression based on the above significant phenotypes. The expression comparison analysis (Figure 3) indicated that expression of CTSH mRNA was relatively lower in sample type of solid tissue normal (all $P<0.001)$. For histological type, classical patients showed higher CTSH mRNA expression (all $P<0.05$ ). Higher expression of CTSH mRNA expression was also observed in T3 and N1 stage (all $P<0.05$ ). In terms of tumor stage, patients in stage III presented lower expression of CTSH mRNA expression (all $P<0.01$ ).

In addition, we evaluated the impacts of CTSH methylation and copy number variation $(\mathrm{CNV})$ on its mRNA expression level. The analysis showed that CTSH mRNA expression was negatively related to methylation level (Figure 4A, $P<0.001$ ), but positively correlated with CTSH CNV (Figure 4B, $P<0.001$ ). Further, we found that CTSH methylation level in THCA was lower than that in normal patients (Figure $4 \mathrm{C}, P<0.001$ ). An integral survival analysis showed no correlation between methylation status and survival time (Figure 4D). But when we only controlled age and gender, a correlation between
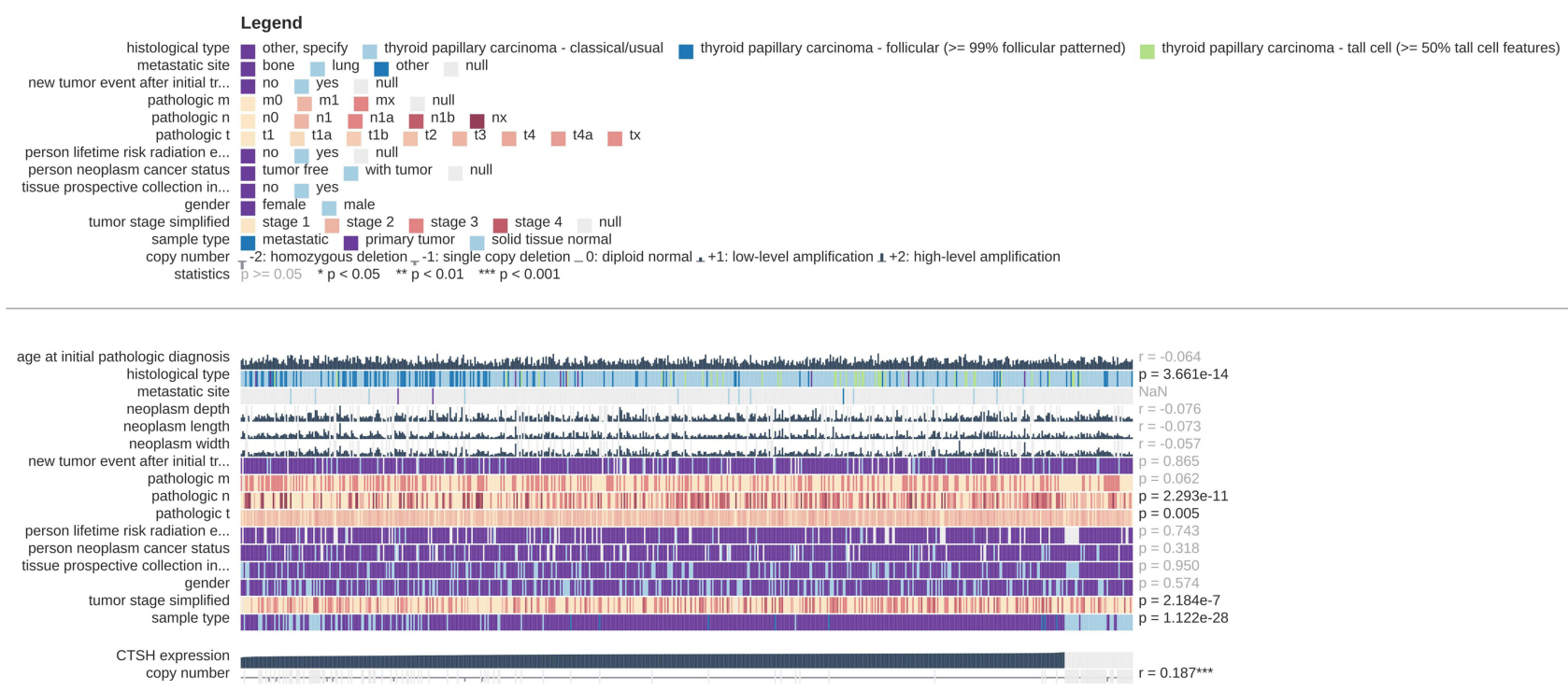

Figure 2 Association between CTSH mRNA expression and clinical data of THCA patients $(n=568)$. From top to bottom, it was the legend, the clinical data, the expression and the copy number data. Samples are arranged from left to right. The samples are ordered by the expression of CTSH. So in this case, samples with low CTSH expression are located on the left side of the plot, whereas samples with high CTSH expression are on the right. $* P<0.05, * * P<0.01$ and $* * * P<0.00 \mathrm{I}$. 


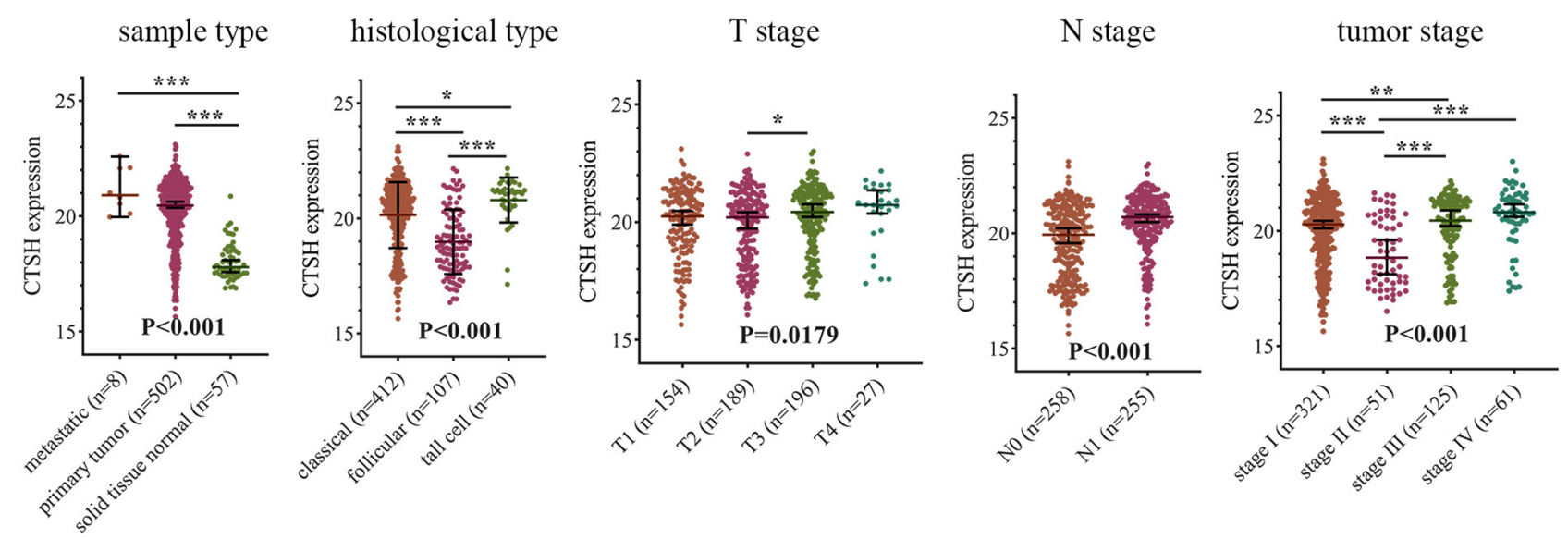

Figure 3 Association between CTSH mRNA expression and significant clinical features in THCA. The data was presented as median and $95 \% \mathrm{Cl}$ (black line). $* P<0.05$ and $* * P<0.01$ and $* * * P<0.001$. The one-way ANOVA was used to compare differences among groups, followed by post hoc multiple comparison.

Abbreviation: $\mathrm{Cl}$, confidence interval; T, primary tumor; TI, small, minimally invasive within primary organ site; T2, larger, more invasive within the primary organ site; $\mathrm{T} 3$, larger and/or invasive beyond margins of primary organ site; T4, very large and/or very invasive, spread to adjacent organs. N, regional lymph nodes; N0, no regional lymph node metastasis; NI, metastases in I to 3 axillary lymph nodes.

methylation status and prognosis was observed (Figure 4E). The T, N, M and cancer stage did not influence the survival difference between 2 groups with different methylation levels. The CNV of CTSH did not directly influence the overall survival of THCA patients (Figure 4F).

From Cox regression analysis, we found the significance of immune cell on the patient's survival. We then evaluated the correlation between CTSH mRNA expression and abundance of B cell, CD4 T cell and macrophage. Among B cells, Mem_B cell showed the highest correlation with CTSH mRNA expression. Regarding CD4 T cell, Tcm_CD4 presented the strongest relation with CTSH mRNA expression. The correlation analysis between CTSH mRNA expression and Mem_B cell, Tcm_CD4 cell and macrophage abundance was presented in Figure 5.

\section{The Expression of CTSH in Normal and Cancer Tissues}

Through HPA database analysis, we found that mRNA expression of CTSH has characteristic of tissue specificity, and CTSH expression was obviously enhanced in the tissue of lung (Figure 6A). In addition, mRNA expression was also enriched in lymphoid tissues such as lymphoid node and tonsil. The CTSH protein expression exerted no tissue specificity (Figure 6B).

Besides the expression overview in normal tissue, the abnormal expression of CTSH in cancer tissues was also assessed. The above RNA expression overview indicated the low cancer specificity of CTSH (Figure 7A), and relatively higher expression was observed in thyroid cancer, lung cancer and urothelial cancer. The protein expression overview (Figure 7B) suggested that higher expression appeared in prostate cancer, thyroid cancer and renal cancer. Among human cancers, both mRNA and protein expressions of CTSH in thyroid cancer was relatively higher than that in other cancer types.

\section{The Genetic Alteration of CTSH in THCA}

The cBioPortal database contained 397 samples with THCA, and $3 \%$ of patients presented the genetic alteration of CTSH. The mutation site was located at protein domain I233Nfs*3 (Figure 8A). The mRNA high expression showed the highest alteration frequency in both papillary and follicular thyroid cancer (Figure 8B). The main mutation type of $\mathrm{CTSH}$ in thyroid carcinoma was missense substitution $(75.00 \%)$ (Figure $8 \mathrm{C}$ ). The nucleotide mutation was mainly presented as $\mathrm{G}>\mathrm{T}(50.00 \%)$ (Figure $8 \mathrm{D})$.

\section{Enrichment Analysis}

In order to obtain a more in-depth understanding of the CTSH in THCA, we determined 206 co-expressed genes playing similar function with $\mathrm{CTSH}$ as setting the threshold of $P<0.001$ and absolute Spearman correlation $>0.6$. GO annotation was then performed. The GO terms after clustering were significantly enriched in 20 terms (Figure 9A), such as actin cytoskeleton organization, regulation of hormone levels, positive regulation of transferase activity and regulation of membrane potential. To 
A

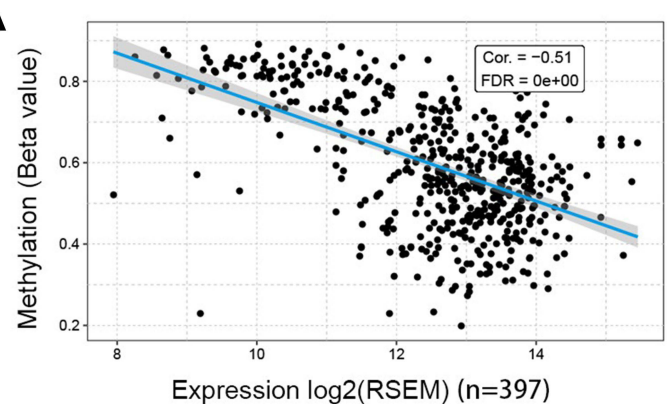

B

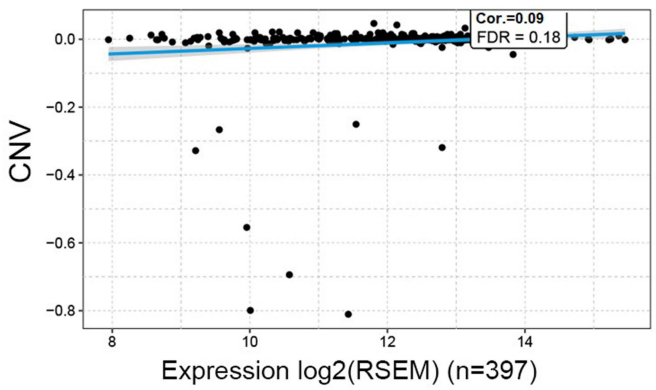

C

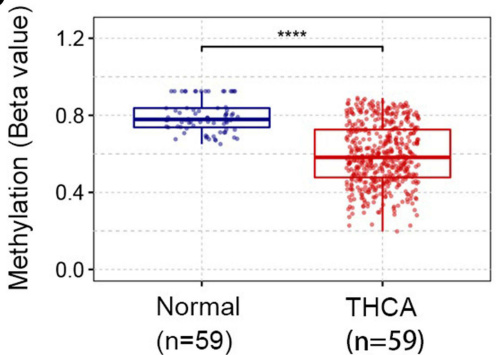

E

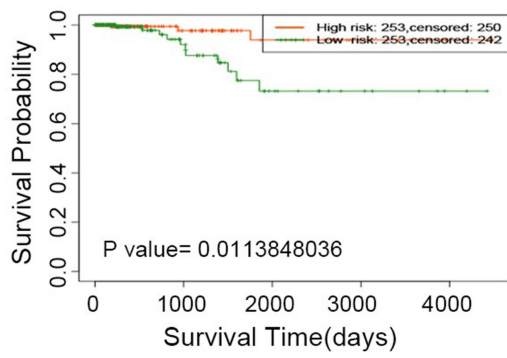

D
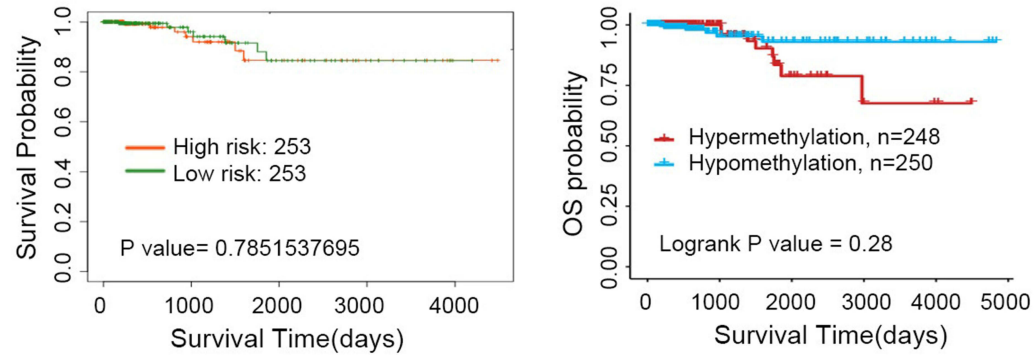

F

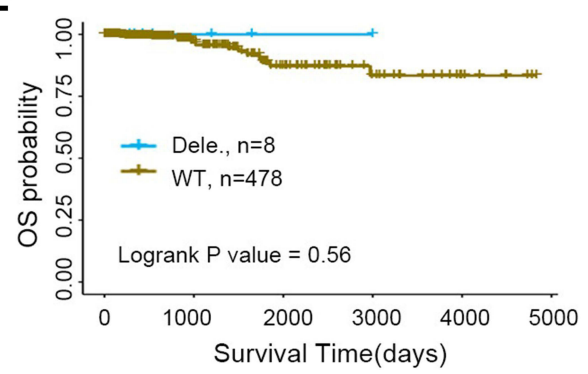

Figure 4 Expression and prognosis analyses on CTSH in THCA. (A) Spearman correlation between CTSH mRNA expression and methylation. (B) Spearman correlation between CTSH mRNA expression and copy number variation (CNV). (C) Methylation level of CTSH in normal and THCA tissues. $* * * * P<0.000 \mathrm{I}$. (D) Integral survival analysis about effect of CTSH methylation level on overall survival (OS) of THCA patients. (E) The effect of CTSH methylation level on overall survival of THCA patients after controlling age and gender parameters. (F) The effect of CTSH CNV on OS of THCA patients. The method of Spearman correlation analysis was used for data of ( $\mathbf{A}$ and $\mathbf{B})$. Data in $\mathbf{C}$ was compared with independent T-test. Kaplan-Meier and logrank test were used for compare survival time in (D, $\mathbf{E}$ and $\mathbf{F}$ ).
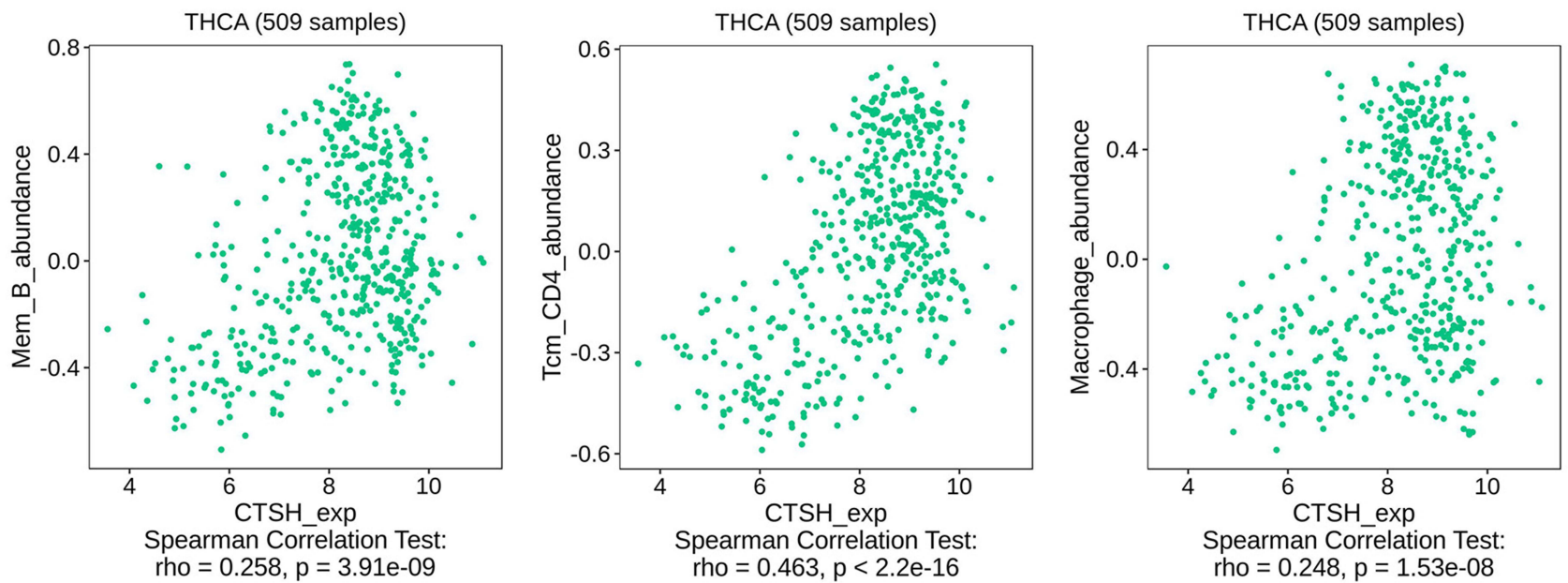

Figure 5 Association between CTSH mRNA expression and infiltration level of immune cells. Mem_B: Memory B cell. Tcm_CD4: Central memory CD4 T cell. Spearman correlation analysis was used to present relationship between 2 variables. 

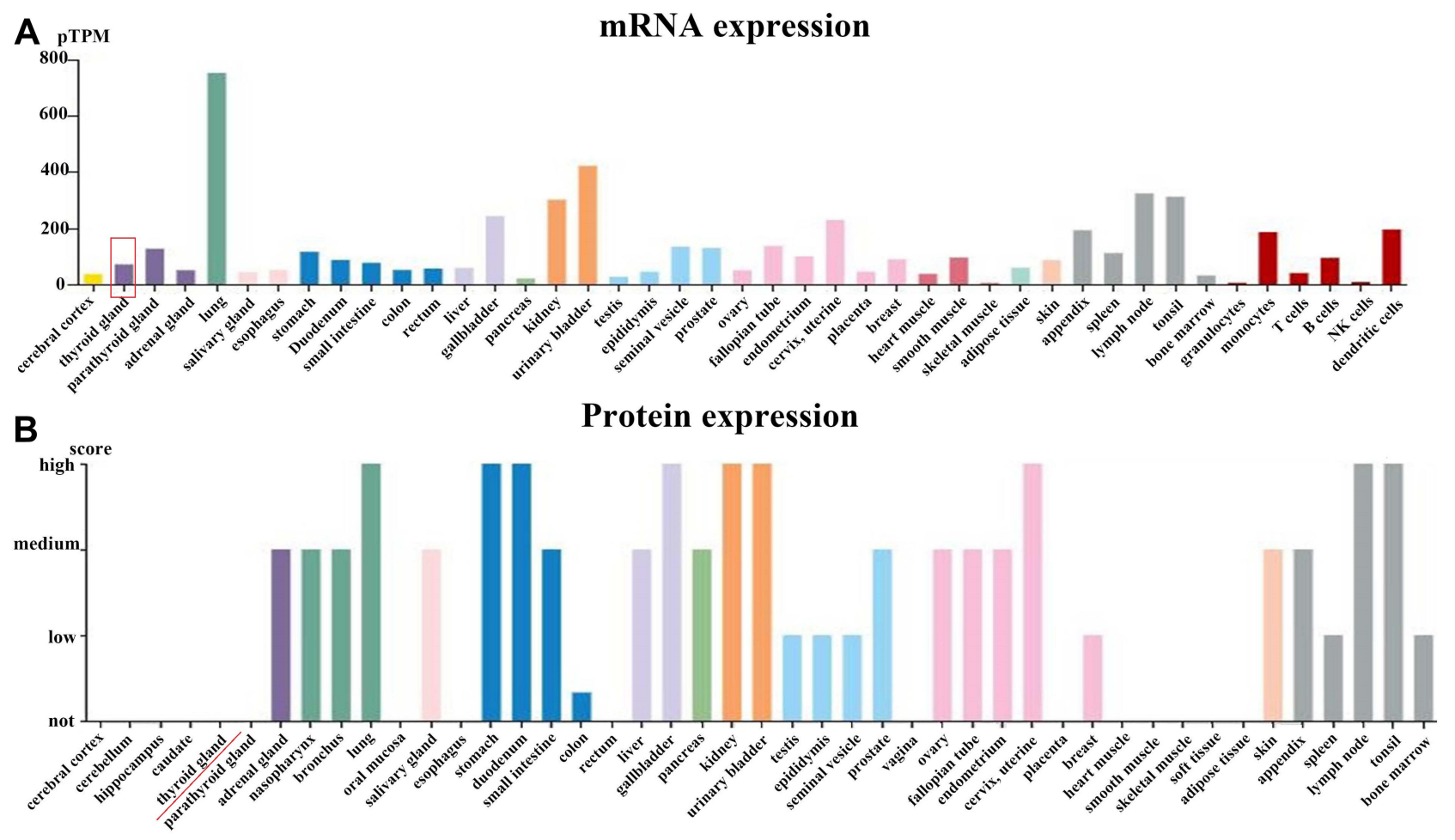

Figure 6 Expression overview of CTSH in normal tissues. (A) mRNA expression. (B) Protein expression. Red box indicated thyroid gland.

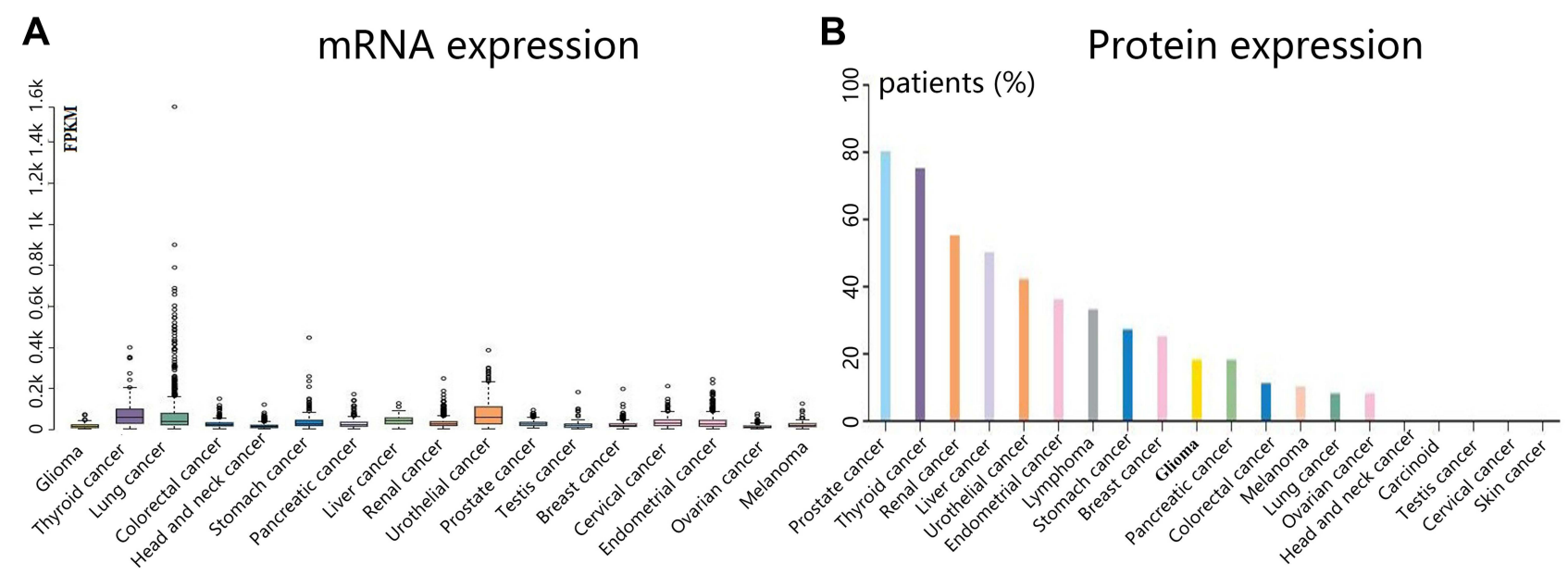

Figure 7 Expression overview of CTSH in human cancers. (A) mRNA expression. (B) Protein expression. Red box indicated thyroid cancer.

systematically clarify the key pathways involved in THCA pathogenesis, pathway enrichment analysis was subsequently carried out. KEGG pathway enrichment analysis showed that these co-expressed genes were significantly enriched in hedgehog signaling pathway, thyroid hormone synthesis, adherens junction and apelin signaling pathway (Figure 9B).

Next, GSEA analysis on CTHS involved in THCA was performed to explore significant pathways. A total of 43 significant pathways were detected containing 28 positive correlated and 15 negative correlated pathways with CTSH. Because of favorable impacts of CTSH high expression on the survival of patients, we just focused on positive related pathways of CTSH. The analysis in Figure 10 showed that CTSH positively correlated with pathways of natural killer mediated cytotoxicity, cytokinecytokine receptor interaction and JAK-STAT signaling pathway. In addition, we found that hedgehog signaling 
A

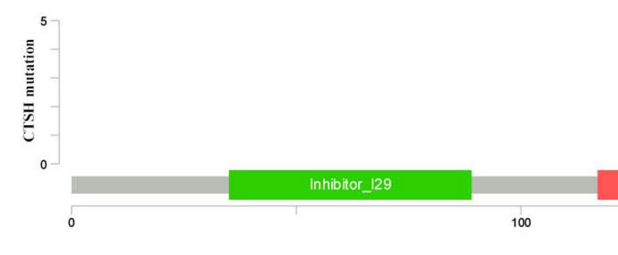

B

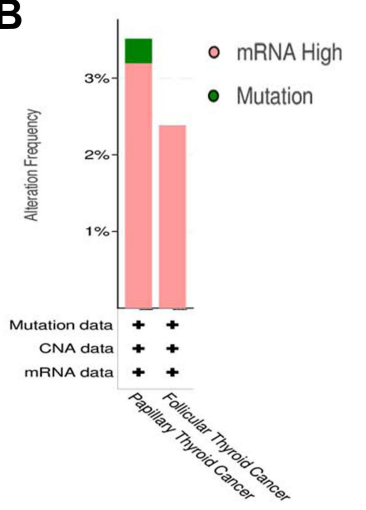

C

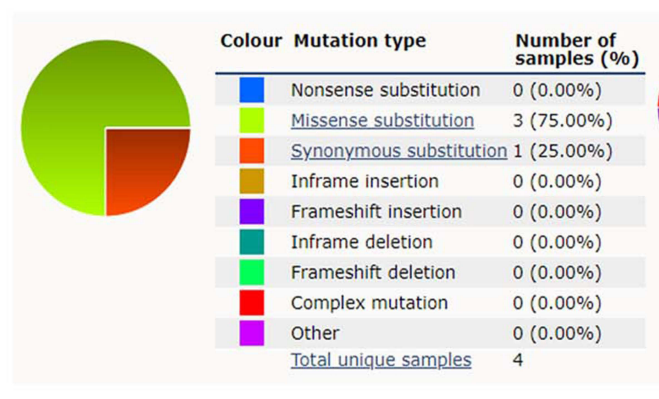

I233Nfs*3

200

D

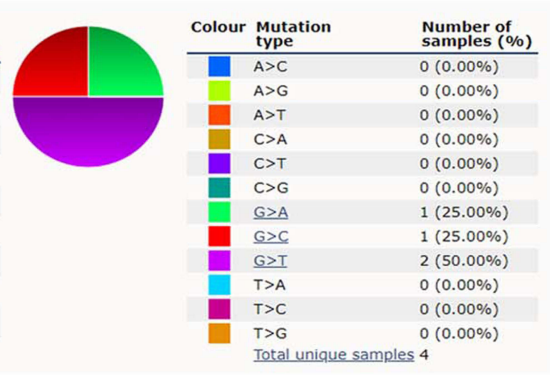

Figure 8 The genetic alteration of CTSH in THCA. (A) Mutation sites. (B) Alteration frequency based on the cancer types. (C) Mutation type. (D) Nucleotide mutation. The data of A and B was obtained from Thyroid Carcinoma (TCGA, Firehose Legacy) dataset ( $n=397)$ in cBioportal database. Data of C and D was obtained from COSMIC database.

pathway was upregulated in CTSH low expression group, indicating a negative correlation between CTSH and hedgehog pathway.

\section{PPI Network Analysis}

The String online database was employed to construct the PPI network of co-expressed genes, then, the Cytoscape software was used to visualize interactions network. After analysis, there were 120 nodes and 134 edges in PPI network, and the most significant networks were presented in Figure 11A. From the PPI network, the top 10 nodes ranked by MCC method were determined (Figure 11B). The top 10 genes and corresponding score were as follows: TIMP1 (35), PRKACB (27), MFGE8 (27), SERPINA1 (27), ADCY8 (26), GNAI1 (24), FAM20C (24), MXRA8 (24), ANXA1 (18) and ITPR1 (16). Finally, we explored the correlation between CTSH and 10 hub genes involved in THCA by adjusting the patient's age. Association analysis (Figure 12) showed that expression of 7 hub genes was positively related to CTSH expression, while 3 hub genes negatively correlated with CTSH expression.

\section{Discussion}

Cathepsins have recently emerged as enzymes that were active in cancer development. However, there was no report on the role of cathepsin $\mathrm{H}$ (CTSH) in THCA. In the present study, we initially evaluated the expression of CTSH in THCA patients. Our results showed that CTSH was highly expressed in THCA patients compared with the normal group. CTSH expression correlated with the cancer type, $\mathrm{N}$ stage and clinical stage of THCA patients. In addition, CTSH expression was positively associated with the prognosis of patients with THCA, and high expression of CTSH improved the prognosis in terms of overall survival. Several researches have reported the favorable role of CTSH in human cancers. The study suggested that high expression of CTSH correlated with a better outcome and increased the survival time of patients with head and neck carcinoma. ${ }^{14}$ The low expression of CTSH was not conducive to the prognosis and led to a highest risk of death in patients with colorectal cancer. ${ }^{15}$ Inversely, other investigations showed the undesirable effects of CTSH in cancers. Previous study showed the significant difference of CTSH level between lung tumor and non-tumorous tissues. ${ }^{16}$ This study further indicated that the difference was associated with the cigarette smoking status, and smokers with high level of CTSH experienced poor survival. In addition, the expression of CTSH significantly increased in melanoma patients as well, and metastatic melanoma patients with high contents of CTSH experienced shorter overall 

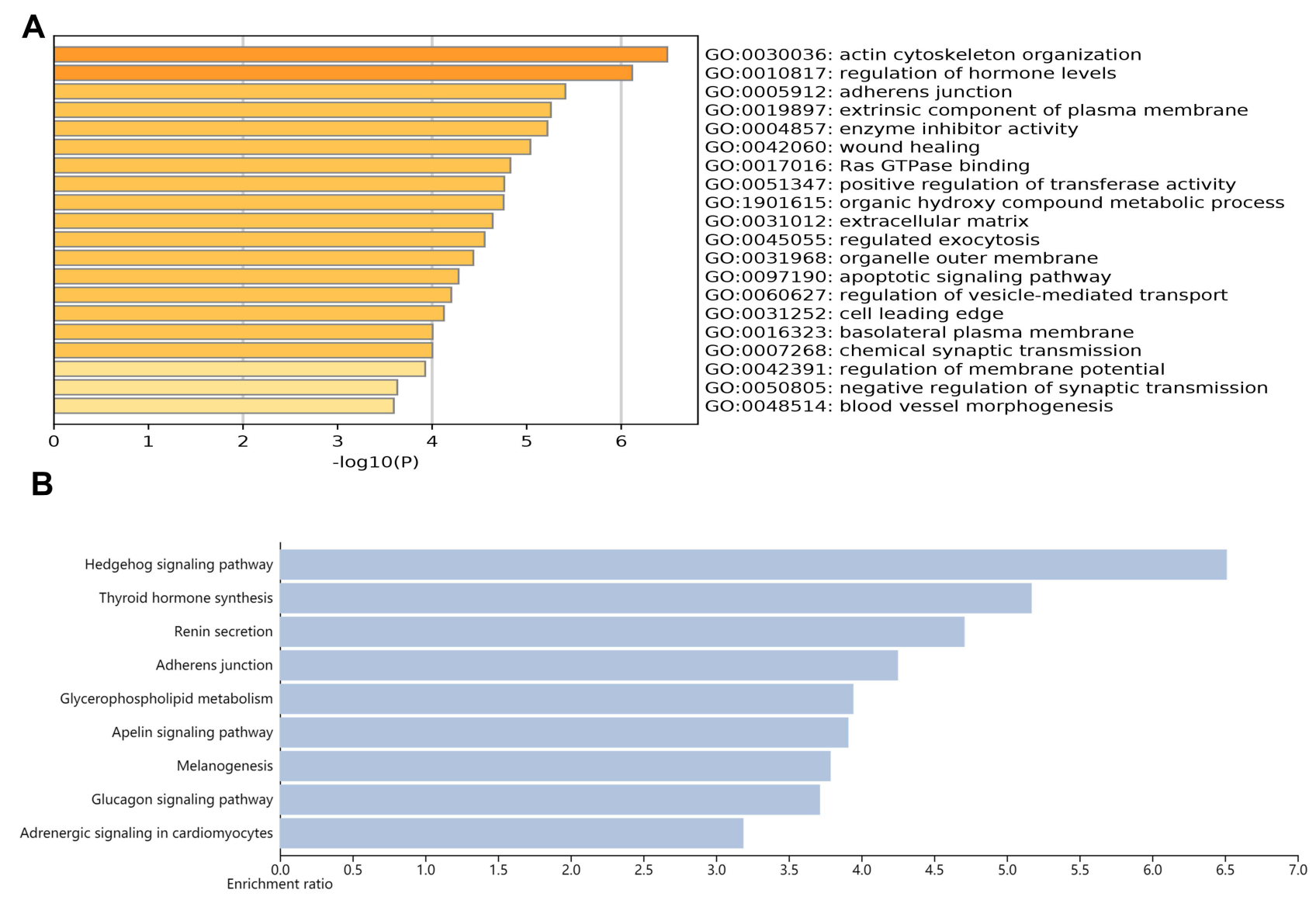

Figure 9 Functional enrichment analysis. (A) Significant GO terms predicted in Metascape database. (B) Important KEGG pathway predicted in WebGestalt database. Abbreviations: GO, Gene Ontology; KEGG, Kyoto Encyclopedia of Genes and Genomes.

Natural killer cell mediated cytotoxicity

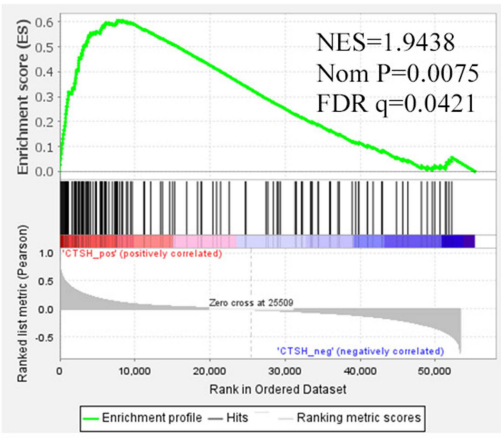

Cytokine cytokine receptor interaction

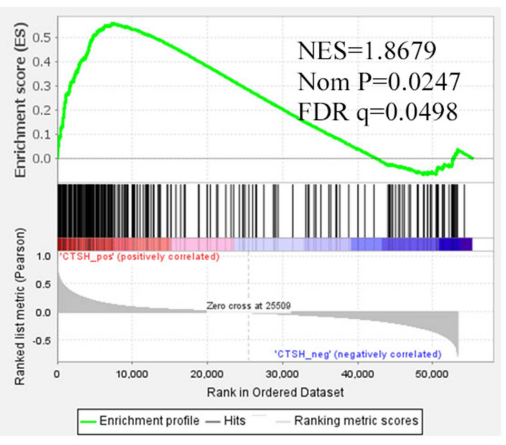

Jak Stat signaling pathway

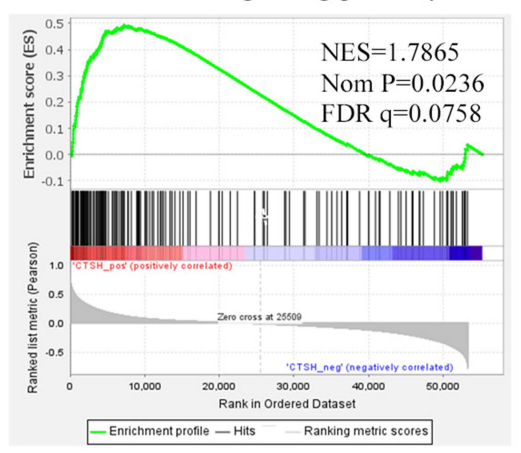

Figure 10 GSEA analysis on CTSH in THCA. The line indicated the enrichment score. The part below horizontal axis presented the individual genes associated with this gene set. Vertical axis showed the enrichment score. Peak value of line chart indicated the enrichment score of gene set. The genes in front of peak value were regarded as the core genes in this gene set. The peak value was located at red section, which indicated that the genes set was positively associated with one gene expression. While located in blue section, it indicated genes set was negatively associated with one gene expression.

survival rates. ${ }^{17}$ All these findings revealed that CTSH could be identified as a significant prognostic factor for patients with cancers.

CTSH has been considered to play a vital role in tumor progression. CTSH-mediated processing of talin might promote cancer cell progression by affecting integrin activation and adhesion strength. ${ }^{18} \mathrm{CTSH}$ could indirectly regulate the bone morphogenetic protein-4 (BMP-4) in various human cell lines. ${ }^{19}$ Moreover, up-regulation of CTSH not only promoted the invasion of tumor cells by 
A

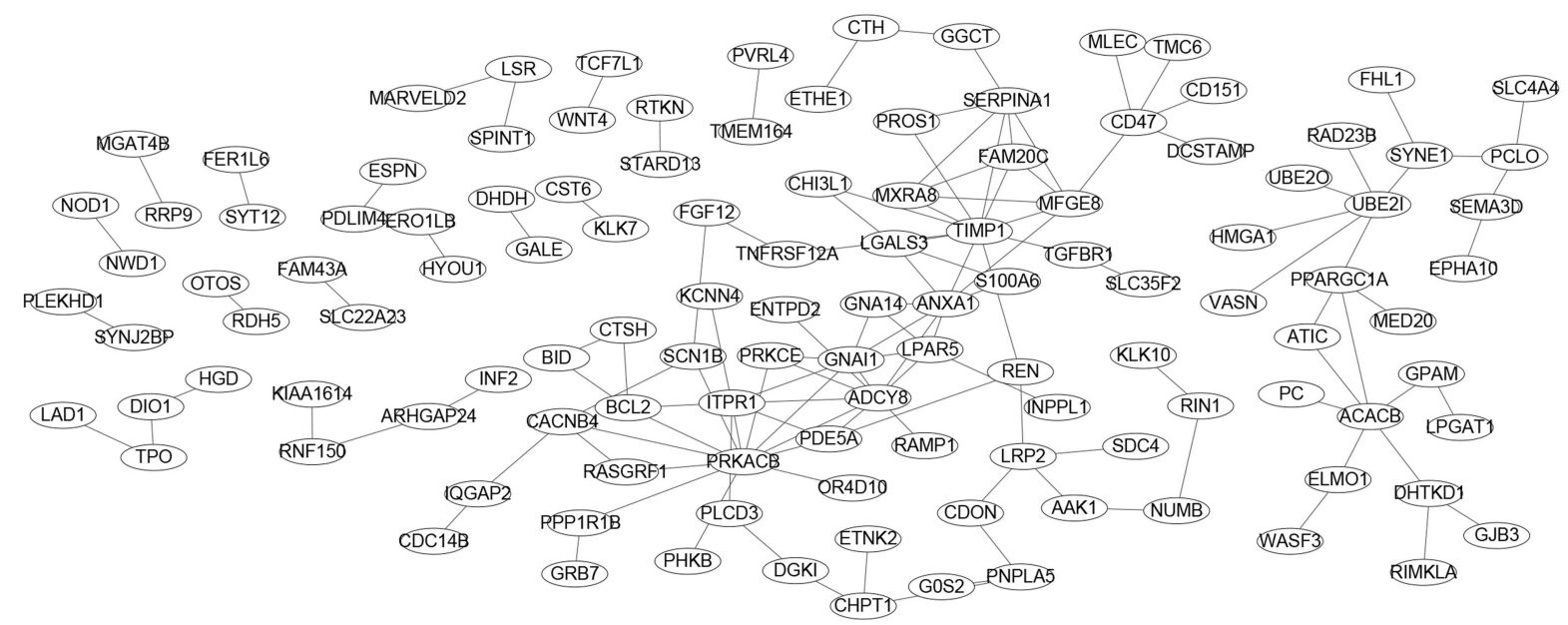

B
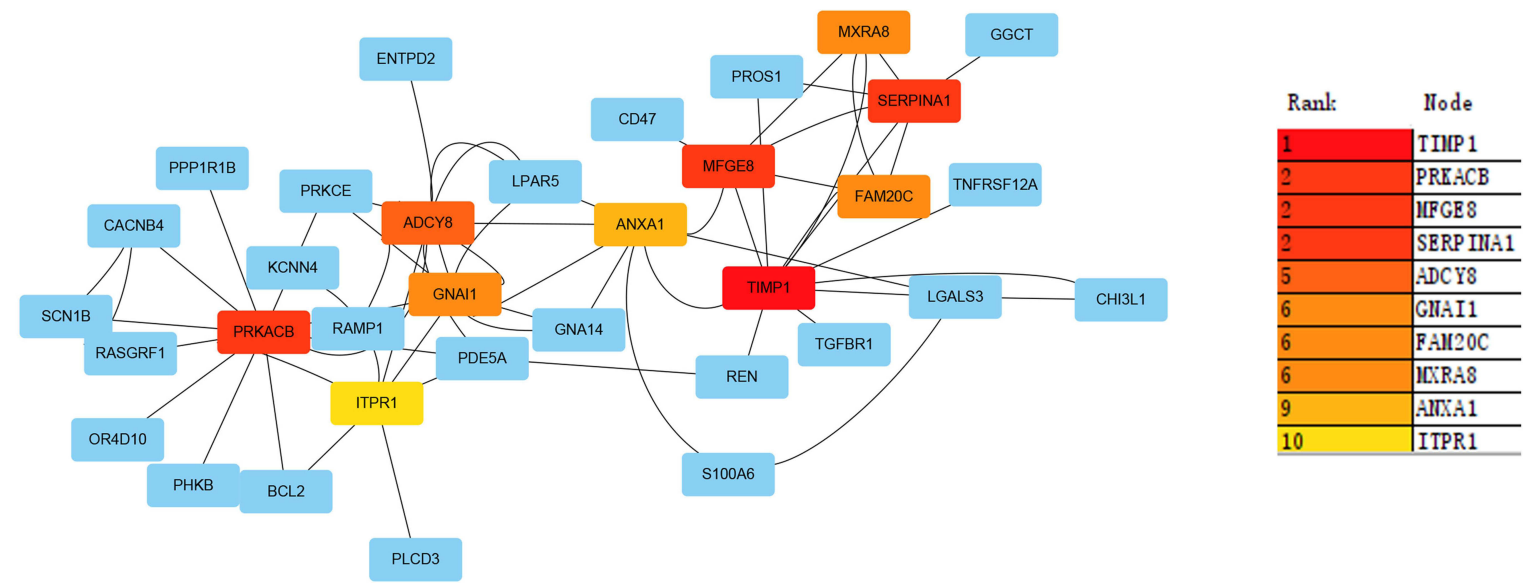

Figure II The protein-protein interaction (PPI) networks. (A) PPI network visualization. (B) Top 10 hub genes ranked by score using MCC method. The hub gene was highlighted with red color, and the node with blue was related genes associated with hub genes.

degradation of the extracellular matrix,,$^{20}$ also increased the cell migration by activation of the extracellular signalregulated kinase. ${ }^{21}$ Another study demonstrated the important tumor-promoting roles for CTSH using mouse model of human cancer, ${ }^{22}$ which indicated that deletion of CTSH significantly impaired the angiogenic switching and resulted in a reduction in the subsequent number of tumors that formed. It followed that CTSH participated in the human cancers through various processes.

In this study, we also performed the enrichment analysis to reveal the potential role of CTSH involved in THCA. Our results indicated that CTSH was significantly associated with the apelin and hedgehog signaling pathway. Unfortunately, no studies reported the correlation between CTSH expression and these two pathways up to now. A recent study showed that apelin inhibition could prevent resistance and metastasis associated with antiangiogenic therapy. ${ }^{23,24}$ Apelin was associated with the promotion of hepatocellular carcinoma through activating PI3K/Akt pathway and was a druggable target. ${ }^{25}$ In addition, apelin can induce the lymphangiogenesis and, accordingly, play an important role in lymphatic tumor progression. ${ }^{26}$ This study opened the door for the development of novel anticancer therapies targeting lymphangiogenesis. In addition, Apelin has known pleiotropic effects on the immune microenvironment. The Apelin expression can be promoted by Tregs. ${ }^{27}$ When Apelin induction was combined with an anticancer vaccine that boosts the immune system and generates natural killer $\mathrm{T}$ (NKT) cells, the normalized vessels allowed trafficking of NKT cells to the tumor and induced a strong antitumor response. ${ }^{28}$ Hedgehog pathway has been demonstrated to closely correlate with the progression of human cancers. Previous study indicated that hedgehog signaling pathway participated in the EMT and invasion of non-small cell lung carcinoma. ${ }^{29}$ The blockage of hedgehog signaling 


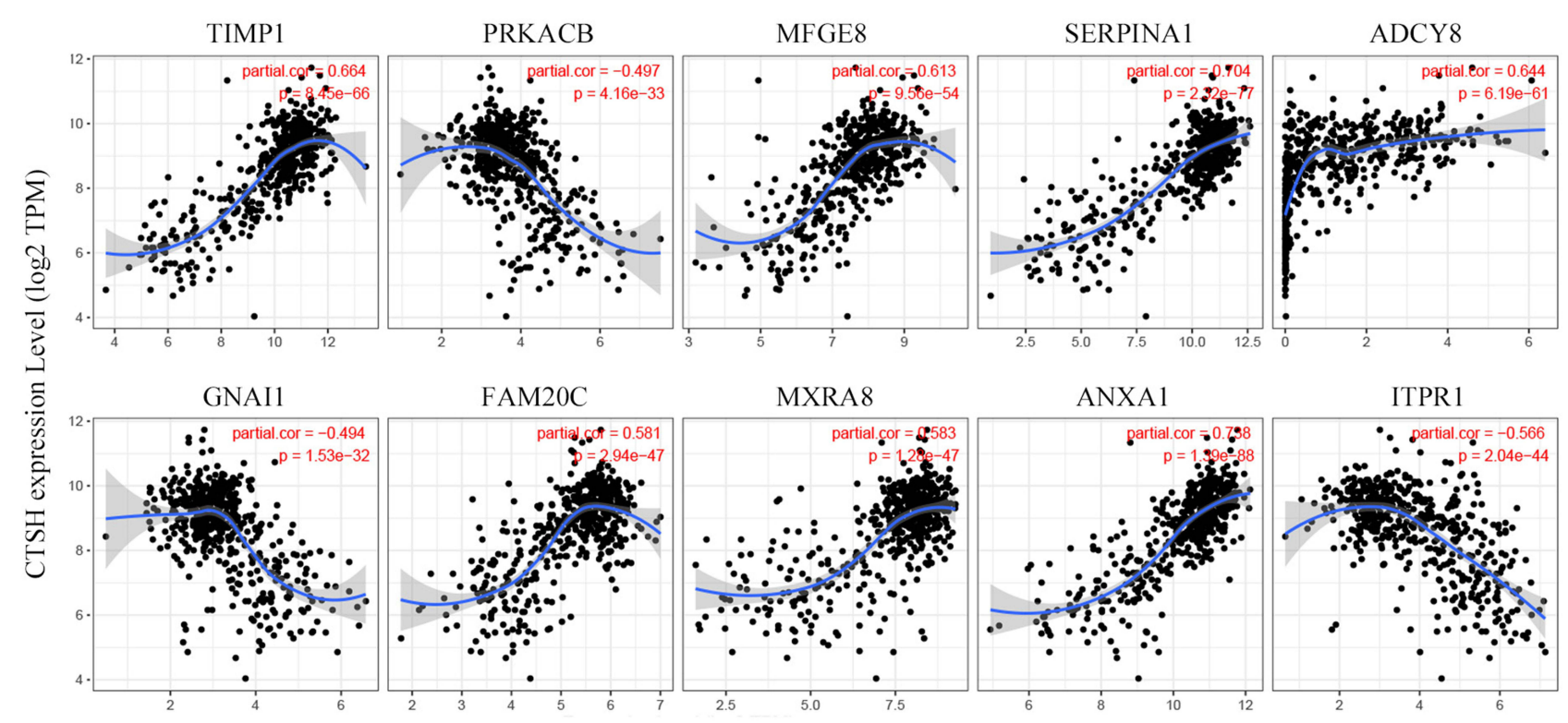

Expression Level (log2 TPM)

Figure 12 Correlation analysis between CTSH and 10 hub genes expression in THCA which was adjusted by patient's age. Horizontal axis indicated mRNA expression of hub genes, and vertical axis referred to CTSH mRNA expression. Spearman correlation analysis was used to present relationship between 2 variables.

promoted the autophagic apoptosis in nasopharyngeal carcinoma. ${ }^{30}$ Moreover, the inhibition of hedgehog signaling pathway induced the apoptosis and impeded the proliferation, migration, and invasion in epithelial ovarian cancer. ${ }^{31}$ In terms of immune system, hedgehog pathway inhibition can alter fibroblast composition and immune infiltration in cancer microenvironment. ${ }^{32}$ Hanna et al identified that hedgehog signaling mediated a molecular crosstalk between cancer cells and macrophages, which culminated in alternative polarization of macrophages. ${ }^{33}$ It followed that Apelin and hedgehog signaling pathways were of great significance in the progression of human cancers.

We finally determined the important hub genes among these co-expressed genes with CTSH. The study indicated that regulation of TIMP1 may be involved in tumor invasion and metastasis in oral cancer, ${ }^{34}$ papillary thyroid cancer ${ }^{35}$ and colon cancer. ${ }^{36}$ MFGE8 could reduce the apoptosis and promote proliferation, ${ }^{37}$ also regulate the pathologic angiogenesis. ${ }^{38}$ ITPR1 was able to induce the autophagy. ${ }^{39}$ GNAI1 can suppress the tumor cell migration and invasion. ${ }^{40}$ It followed that CTSH and these co-expressed genes played a significant role in cancer and were implicated in tumorigenic processes, such as angiogenesis, proliferation, apoptosis and invasion. Although several targets associated with CTSH in THCA were detected, the detailed regulation and interaction between CTSH and hub genes remain unclear, which needs further confirmation through experimental verification.

\section{Conclusion}

This study initially revealed the clinical significance of CTSH in THCA. High expression of CTSH was found in THCA, which was positively associated with overall survival of patients. CTSH was further determined as an independent prognostic factor in THCA. Several factors exerted potential regulation effects on CTSH mRNA expression including clinical characteristics, copy number, methylation level and other genes. We initially found CTSH might correlate with hedgehog signaling pathway and JAK-STAT signaling pathway, and detailed regulation should be further verified by series of functional analyses. The present work provided a new molecular biomarker for THCA treatment. However, further studies are still needed to explore the specific molecular mechanisms of CTSH for THCA initiation and progression in the future.

\section{Data Available Statement}

The datasets used and/or analyzed during the current study are available from the corresponding author on reasonable request.

\section{Funding}

There is no funding to report. 


\section{Disclosure}

These authors have no conflicts of interest to declare.

\section{References}

1. Siegel RL, Miller KD, Jemal A. Cancer statistics, 2018. CA Cancer J Clin. 2018;68:7-30. doi:10.3322/caac.21442

2. McGuire S. World Cancer Report 2014. Geneva, Switzerland: world Health Organization, International Agency for Research on Cancer, WHO Press, 2015. Adv Nutr. 2016;7:418-419. doi:10.3945/ an. 116.012211

3. Ramsey S, Blough D, Kirchhoff A, et al. Washington State cancer patients found to be at greater risk for bankruptcy than people without a cancer diagnosis. Health Aff. 2013;32(6):1143-1152. doi:10.1377/hlthaff.2012.1263

4. Randle RW, Bushman NM, Orne J, et al. Papillary thyroid cancer: the good and bad of the "good cancer". Thyroid. 2017;27:902-907. doi:10.1089/thy.2016.0632

5. Pemayun TG. Current Diagnosis and management of thyroid nodules. Acta Med Indones. 2016;48:247-257.

6. Im B, Kominami E, Grube D, Uchiyama Y. Immunocytochemical localization of cathepsins $\mathrm{B}$ and $\mathrm{H}$ in human pancreatic endocrine cells and insulinoma cells. Histochemistry. 1989;93:111-118. doi:10.1007/BF00315963

7. Soderstrom M, Ekfors T, Bohling T, et al. Cysteine proteinases in chondrosarcomas. Matrix Biol. 2001;19(8):717-725. doi:10.1016/ S0945-053X(00)00124-4

8. Soond SM, Kozhevnikova MV, Zamyatnin AA. 'Patchiness' and basic cancer research: unravelling the proteases. Cell Cycle. 2019;18:1687-1701. doi:10.1080/15384101.2019.1632639

9. Soond SM, Kozhevnikova MV, Townsend PA, et al. Cysteine Cathepsin Protease Inhibition: an update on its Diagnostic, Prognostic and Therapeutic Potential in Cancer. Pharmaceuticals. 2019;12(2):87. doi:10.3390/ph12020087

10. Del Re EC, Shuja S, Cai J, Murnane MJ. Alterations in cathepsin $\mathrm{H}$ activity and protein patterns in human colorectal carcinomas. $\mathrm{Br}$ J Cancer. 2000;82:1317-1326. doi:10.1054/bjoc.1999.1098

11. Gabrijelcic D, Svetic B, Spaic D, et al. Cathepsins B, H and L in human breast carcinoma. Eur $J$ Clin Chem Clin Biochem. 1992;30:69-74.

12. Waghray A, Keppler D, Sloane BF, et al. Analysis of a truncated form of cathepsin H in human prostate tumor cells. J Biol Chem. 2002;277 (13):11533-11538. doi:10.1074/jbc.M109557200

13. Kos J, Smid A, Krasovec M, et al. Lysosomal proteases cathepsins D, B, H, L and their inhibitors stefins A and B in head and neck cancer. Biol Chem Hoppe Seyler. 1995;376:401-405. doi:10.1515/ bchm3.1995.376.7.401

14. Budihna M, Strojan P, Smid L, et al. Prognostic value of cathepsins B, H, L, D and their endogenous inhibitors stefins A and B in head and neck carcinoma. Biol Chem Hoppe Seyler. 1996;377:385-390. doi:10.1515/bchm3.1996.377.6.385

15. Schweiger A, Christensen IJ, Nielsen HJ, et al. Serum cathepsin H as a potential prognostic marker in patients with colorectal cancer. Int J Biol Markers. 2004;19(4):289-294. doi:10.1177/1724600 80401900406

16. Kos J, Stabuc B, Schweiger A, et al. Cathepsins B, H, and L and their inhibitors stefin A and cystatin $\mathrm{C}$ in sera of melanoma patients. Clin Cancer Res. 1997;3:1815-1822.

17. Schweiger A, Staib A, Werle B, et al. Cysteine proteinase cathepsin $\mathrm{H}$ in tumours and sera of lung cancer patients: relation to prognosis and cigarette smoking. Br J Cancer. 2000;82:782-788. doi:10.1054/ bjoc. 1999.0999

18. Jevnikar Z, Rojnik M, Jamnik P, et al. Cathepsin H mediates the processing of talin and regulates migration of prostate cancer cells. J Biol Chem. 2013;288(4):2201-2209. doi:10.1074/jbc.M112.436394
19. Rojnik M, Jevnikar Z, Mirkovic B, et al. Cathepsin H indirectly regulates morphogenetic protein-4 (BMP-4) in various human cell lines. Radiol Oncol. 2011;45(4):259-266. doi:10.2478/v10019-0110034-3

20. Frohlich E, Mohrle M, Klessen C. Cathepsins in basal cell carcinomas: activity, immunoreactivity and mRNA staining of cathepsins B, D, H and L. Arch Dermatol Res. 2004;295:411-421. doi:10.1007/ s00403-003-0449-9

21. Wu SM, Huang YH, Yeh CT, et al. Cathepsin H regulated by the thyroid hormone receptors associate with tumor invasion in human hepatoma cells. Oncogene. 2011;30:2057-2069. doi:10.1038/ onc. 2010.585

22. Gocheva V, Chen X, Peters C, et al. Deletion of cathepsin H perturbs angiogenic switching, vascularization and growth of tumors in a mouse model of pancreatic islet cell cancer. Biol Chem. 2010;391 (8):937-945. doi:10.1515/bc.2010.080

23. Jaiprasart $P$, Dogra $S$, Neelakantan $D$, et al. Identification of signature genes associated with therapeutic resistance to anti-VEGF therapy. Oncotarget. 2020;11(1):99-114. doi:10.18632/oncotarget.27307

24. Uribesalgo I, Hoffmann D, Zhang Y, et al. Apelin inhibition prevents resistance and metastasis associated with anti-angiogenic therapy. EMBO Mol Med. 2019;11:e9266. doi:10.15252/emmm.201809266

25. Chen H, Wong CC, Liu D, et al. APLN promotes hepatocellular carcinoma through activating PI3K/Akt pathway and is a druggable target. Theranostics. 2019;9:5246-5260. doi:10.7150/thno.34713

26. Berta J, Hoda MA, Laszlo V, et al. Apelin promotes lymphangiogenesis and lymph node metastasis. Oncotarget. 2014;5(12):4426-4437. doi:10.18632/oncotarget.2032

27. Leung OM, Li J, Li X, et al. Regulatory T Cells Promote Apelin-Mediated Sprouting Angiogenesis in Type 2 Diabetes. Cell Rep. 2018;24:1610-1626. doi:10.1016/j.celrep.2018.07.019

28. Kidoya H, Kunii N, Naito H, et al. The apelin/APJ system induces maturation of the tumor vasculature and improves the efficiency of immune therapy. Oncogene. 2012;31(27):3254-3264. doi:10.1038/ onc.2011.489

29. Xu H, Dun S, Gao Y, et al. TMEM107 inhibits EMT and invasion of NSCLC through regulating the Hedgehog pathway. Thorac Cancer. 2020;1:87.

30. Chen J, Wen B, Wang Y, et al. Jervine exhibits anticancer effects on nasopharyngeal carcinoma through promoting autophagic apoptosis via the blockage of Hedgehog signaling. Biomed Pharmacother. 2020;132:110898. doi:10.1016/j.biopha.2020.110898

31. Liu Y, Gao S, Zhu J, et al. Dihydroartemisinin induces apoptosis and inhibits proliferation, migration, and invasion in epithelial ovarian cancer via inhibition of the hedgehog signaling pathway. Cancer Med. 2018;7(11):5704-5715. doi:10.1002/cam4.1827

32. Steele NG, Biffi G, Kemp SB, et al. Inhibition of Hedgehog Signaling Alters Fibroblast Composition in Pancreatic Cancer. Clin Cancer Res. 2021;27(7):2023-2037. doi:10.1158/1078-0432.CCR20-3715

33. Hanna A, Metge BJ, Bailey SK, et al. Inhibition of Hedgehog signaling reprograms the dysfunctional immune microenvironment in breast cancer. Oncoimmunology. 2018;8:1548241. doi:10.1080/ 2162402X.2018.1548241

34. Xu G, Wei J, Huangfu B, et al. Animal model and bioinformatics analyses suggest the TIMP1/MMP9 axis as a potential biomarker in oral squamous cell carcinoma. Mol Carcinog. 2020;59:1302-1316. doi: $10.1002 / \mathrm{mc} .23258$

35. Zhang W, Sun W, Qin Y, et al. Knockdown of KDM1A suppresses tumour migration and invasion by epigenetically regulating the TIMP1/MMP9 pathway in papillary thyroid cancer. $J$ Cell Mol Med. 2019;23:4933-4944. doi:10.1111/jcmm.14311

36. Song G, Xu S, Zhang H, et al. TIMP1 is a prognostic marker for the progression and metastasis of colon cancer through FAK-PI3K/AKT and MAPK pathway. J Exp Clin Cancer Res. 2016;35:148. doi:10.1186/s13046-016-0427-7 
37. Li H, Zhang T, Wang $\mathrm{K}$, et al. MFGE8 protects against $\mathrm{CCl} 4$ induced liver injury by reducing apoptosis and promoting proliferation of hepatocytes. J Cell Physiol. 2019;2:587.

38. Motegi S, Leitner WW, Lu M, et al. Pericyte-derived MFG-E8 regulates pathologic angiogenesis. Arterioscler Thromb Vasc Biol. 2011;31:2024-2034. doi:10.1161/ATVBAHA.111.232587

39. Messai Y, Noman MZ, Hasmim M, et al. ITPR1 protects renal cancer cells against natural killer cells by inducing autophagy. Cancer Res. 2014;74:6820-6832. doi:10.1158/0008-5472.CAN-14-0303
40. Yao J, Liang LH, Zhang Y, et al. GNAI1 Suppresses Tumor Cell Migration and Invasion and is Post-Transcriptionally Regulated by Mir-320a/c/d in Hepatocellular Carcinoma. Cancer Biol Med. 2012;9:234-241.

\section{Publish your work in this journal}

The International Journal of General Medicine is an international, peer-reviewed open-access journal that focuses on general and internal medicine, pathogenesis, epidemiology, diagnosis, monitoring and treatment protocols. The journal is characterized by the rapid reporting of reviews, original research and clinical studies across all disease areas. The manuscript management system is completely online and includes a very quick and fair peer-review system, which is all easy to use. Visit http://www.dovepress.com/ testimonials.php to read real quotes from published authors.

Submit your manuscript here: https://www.dovepress.com/international-journal-of-general-medicine-journal 